\title{
ERROR ANALYSIS ASSOCIATED WITH UNIFORM HERMITE INTERPOLATIONS OF BANDLIMITED FUNCTIONS
}

\author{
Mahmoud H. Annaby and Rashad M. Asharabi
}

\begin{abstract}
We derive estimates for the truncation, amplitude and jitter type errors associated with Hermite-type interpolations at equidistant nodes of functions in Paley-Wiener spaces. We give pointwise and uniform estimates. Some examples and comparisons which indicate that applying Hermite interpolations would improve the methods that use the classical sampling theorem are given.
\end{abstract}

\section{Introduction}

The classical sampling theorem of Whittaker, Kotel'nikov, and Shannon (WKS), plays a major role in communication theory as well as in many applications in approximation theory. The WKS sampling theorem states that if $f(t)$ is any $L^{2}(\mathbb{R})$-function whose Fourier transform has a compact support $[-\sigma, \sigma], \sigma>0$, then it can be reconstructed from its values at $n \pi / \sigma, n \in \mathbb{Z}$ via

$$
f(t)=\sum_{n=-\infty}^{\infty} f\left(\frac{n \pi}{\sigma}\right) S_{n}(t), \quad t \in \mathbb{R}
$$

where

$$
S_{n}(t):= \begin{cases}\frac{\sin (\sigma t-n \pi)}{(\sigma t-n \pi)}, & t \neq \frac{n \pi}{\sigma}, \\ 1, & t=\frac{n \pi}{\sigma} .\end{cases}
$$

The convergence is uniform and absolute on $\mathbb{R}$. See the interesting surveys $[15,12,13]$ for history, references, generalizations and applications in both mathematics and signal analysis. The class of all $L^{2}(\mathbb{R})$-functions whose Fourier transforms vanish outside $[-\sigma, \sigma]$ is known in communication theory as the class of bandlimited signals. Equivalently, see [7, 26], the class of bandlimited

Received April 1, 2009.

2000 Mathematics Subject Classification. 30D10, 41A05, 41A30, 94A20.

Key words and phrases. Hermite interpolations, derivative sampling theorem, truncation, amplitude and jitter errors. 
functions (signals) is the Paley-Wiener space of entire $L^{2}(\mathbb{R})$-functions of exponential type $\sigma$. It will be denoted by $P W_{\sigma}^{2}$. The positive number $\sigma$ is called in engineering terminology the band-width. Thus Expansion (1.1) can be extended for $t \in \mathbb{C}$ with absolute convergence on $\mathbb{C}$ and uniform convergence on compact sets of the complex plane as well as in the $L^{2}(\mathbb{R})$-norm. Expansion is a Lagrange-type interpolation expansion, which has been used extensively in approximation theory, see e.g. [24, 29, 30]. Several types of errors are connected with the use of (1.1). Frequently, the following three types normally appear in the use of the sinc-method:

- The truncation error. This error results from the truncating infinite series (1.1). For a positive integer $N$, this error is defined to be

$$
R_{N}(t):=f(t)-\sum_{|n| \leq N} f\left(\frac{n \pi}{\sigma}\right) S_{n}(t)=\sum_{|n|>N} f\left(\frac{n \pi}{\sigma}\right) S_{n}(t), t \in \mathbb{R} .
$$

- The amplitude error. This error results from using alternate samples $\widetilde{f}(n \pi / \sigma)$ instead of the (unavailable) exact ones $f(n \pi / \sigma)$. The resulting error will be

$$
\mathcal{E} f(t):=\sum_{n=-\infty}^{\infty}\left\{f\left(\frac{n \pi}{\sigma}\right)-\widetilde{f}\left(\frac{n \pi}{\sigma}\right)\right\} S_{n}(t), \quad t \in \mathbb{R} .
$$

- The jitter error. This error arises in case alternate nodes $t_{n}, n \in \mathbb{Z}$, are taken instead of the exact nodes $n \pi / \sigma$. In such case this error has the form

$$
\eta f(t):=\sum_{n=-\infty}^{\infty}\left\{f\left(\frac{n \pi}{\sigma}\right)-f\left(t_{n}\right)\right\} S_{n}(t), \quad t \in \mathbb{R} .
$$

The occurrence of the three types of errors appears in several practical as well as approximation problems. In particular the amplitude and jitter errors appear when the samples or the nodes are not exactly known. But alternate ones can be obtained. In physics, the measured observations are not exactly the ones that fit in the construction of the signals, but they are closer. In numerical analysis, to find numerical approximations of the eigenvalues of boundary value problems, the exact samples can be computed only when the differential equations can be solved in closed forms, which rarely happens. Alternatively, approximate values of the solutions are implemented by using standard numerical methods, like e.g. Runge-Kutta method. It is also known that any desired accuracy $\varepsilon_{n}, \delta_{n}$ could be achieved, subject to the computational prices. See e.g. $[4,5,6]$. There are several studies to estimate these types of errors for bandlimited functions under appropriate conditions. For estimating the truncation error, both pointwise and uniform bounds are given. Piper [27], gave local pointwise bounds of order $N^{-1 / 2}$ for the truncation error; Yao and Thomas [32]; Piper [27]; Brown [9]; gave local bound estimates of order $N^{-1}$ provided that the band-width is shortened. The mostly used result is that of Jagerman [21], for which higher order accuracy under the condition that the truncated bandlimited function 
satisfies smoothness conditions, is derived. To the best of our knowledge, the estimate of $\mathrm{Li}$ [23], gave the first uniform estimate. As for the amplitude and jitter error, Butzer et al. have investigated these errors when the alternate samples and nodes are close to the actual ones, cf. [11, 14, 15].

This paper is concerned with the error analysis on $\mathbb{R}$ associated with Hermitetype interpolation

$$
f(t)=\sum_{n=-\infty}^{\infty}\left\{f\left(\frac{n \pi}{\sigma}\right) S_{n}^{2}(t)+f^{\prime}\left(\frac{n \pi}{\sigma}\right) \frac{\sin (\sigma t-n \pi)}{\sigma} S_{n}(t)\right\}
$$

for bandlimited functions. By a bandlimited function $f$, we mean an element of the Paley-Wiener space $P W_{2 \sigma}^{2}$ of entire $L^{2}(\mathbb{R})$-functions of exponential type $2 \sigma$. The uniform convergence of (1.6) has been established in [20]. More general situations and the absolute convergence are studied by Grozev and Rahman in [17], where not necessarily uniform nodes are considered. Hermite type sampling formulae could be found also in $[19,22]$. In this paper we investigate truncation, amplitude and jitter type-errors associated with (1.6). The aliasing error has been considered by Voss in [31]. For a positive integer $N$ the truncation error associated with (1.6) is defined to be

$$
T(f, N)(t):=\left|f(t)-f_{N}(t)\right|, \quad t \in \mathbb{R}
$$

where

$$
f_{N}(t):=\sum_{|n| \leq N}\left\{f\left(\frac{n \pi}{\sigma}\right) S_{n}^{2}(t)+f^{\prime}\left(\frac{n \pi}{\sigma}\right) \frac{\sin (\sigma t-n \pi)}{\sigma} S_{n}(t)\right\}
$$

We will derive a pointwise estimate for $T(f, N)$ on intervals of $\mathbb{R}$ as well as a uniform estimate on $\mathbb{R}$ giving the Hermite counterpart of the results of Jagerman [21] and $\mathrm{Li}$ [23] established for the classical sampling theorem. As for the amplitude error, let us take the alternate samples $\widetilde{f}\left(\frac{n \pi}{\sigma}\right), \widetilde{f}^{\prime}\left(\frac{n \pi}{\sigma}\right), n \in \mathbb{Z}$ with $\varepsilon_{n}:=f\left(\frac{n \pi}{\sigma}\right)-\tilde{f}\left(\frac{n \pi}{\sigma}\right), \varepsilon_{n}^{\prime}:=f^{\prime}\left(\frac{n \pi}{\sigma}\right)-\tilde{f}^{\prime}\left(\frac{n \pi}{\sigma}\right)$. Assume that there is $\varepsilon>0$ such that $\left|\varepsilon_{n}\right|,\left|\varepsilon_{n}^{\prime}\right| \leq \varepsilon, n \in \mathbb{Z}$. The amplitude error for $t \in \mathbb{R}$ is

$$
\begin{aligned}
\mathcal{A}(\varepsilon, f)(t):=\sum_{n=-\infty}^{\infty}\{ & \left(f\left(\frac{n \pi}{\sigma}\right)-\widetilde{f}\left(\frac{n \pi}{\sigma}\right)\right) S_{n}^{2}(t) \\
& \left.+\left(f^{\prime}\left(\frac{n \pi}{\sigma}\right)-\tilde{f}^{\prime}\left(\frac{n \pi}{\sigma}\right)\right) \frac{\sin (\sigma t-n \pi)}{\sigma} S_{n}(t)\right\} .
\end{aligned}
$$

The time-jitter error arises when the sampling nodes $n \pi / \sigma, n \in \mathbb{Z}$ are replaced by closer ones. In other words for $\delta>0$ and $\left|\delta_{n}\right|,\left|\delta_{n}^{\prime}\right| \leq \delta, n \in \mathbb{Z}$, the 
time-jitter error, $J(\delta, f)$, associated with (1.6) is defined to be

$$
\begin{aligned}
J(\delta, f)(t):=\sum_{n=-\infty}^{\infty}\{ & \left(f\left(\frac{n \pi}{\sigma}\right)-f\left(\frac{n \pi}{\sigma}+\delta_{n}\right)\right) S_{n}^{2}(t) \\
& \left.+\left(f^{\prime}\left(\frac{n \pi}{\sigma}\right)-f^{\prime}\left(\frac{n \pi}{\sigma}+\delta_{n}^{\prime}\right)\right) \frac{\sin (\sigma t-n \pi)}{\sigma} S_{n}(t)\right\} .
\end{aligned}
$$

The goal of this paper is to study the estimates for the truncation, amplitude and jitter type errors defined above. In the next section we give some preliminary results which we will use in the sequel. The estimates of truncation, amplitude and jitter errors associated with (1.6) will be established in Sections 3, 4. The last section contains some numerical comparisons and prospective studies. Now we state some results taken from $[1,2]$ which we will use latter on. First we have the identities [1, pp. 258-259],

$$
\begin{gathered}
\sum_{n=1}^{\infty} \frac{2 k}{n(n+2 k)}=\gamma+\psi(2 k+1), k \geq 0, \quad \sum_{n=1}^{j-1} \frac{1}{n}=\gamma+\psi(j), \quad j \geq 1, \\
\psi(2 k+1)=\psi(2 k)+\frac{1}{2 k}, k \geq 1,
\end{gathered}
$$

where $\gamma$ is the Euler-Mascheroni constant

$$
\gamma:=\lim _{n \rightarrow \infty}\left(\sum_{k=1}^{n} \frac{1}{k}-\log (n)\right),
$$

and $\psi(t)$ is the digamma function, i.e., $\psi(t):=\frac{\Gamma^{\prime}(t)}{\Gamma(t)}$ and $\Gamma(t)$ is the gamma function. It is proved in [2, p. 374], see also [25], that $\psi(t)<\log (t)-\frac{1}{2 t}, t>0$. Thus

$$
\psi(t)<\log (t), \quad t>0
$$

\section{Preliminaries}

In this section we introduce some auxiliary lemmas which we will use in our proofs.

Lemma 2.1. Let $\alpha>0$ and $N \in \mathbb{Z}^{+}:=\{1,2, \ldots\}$. Then for $|t|<N \pi / \sigma$ we have

$$
\sum_{n>N} \frac{1}{(\sigma t-n \pi)^{\alpha+1}}<\frac{1}{\alpha \pi(N \pi-\sigma t)^{\alpha}}
$$

Consequently,

$$
\sum_{n<-N} \frac{1}{(\sigma t-n \pi)^{\alpha+1}}<\frac{1}{\alpha \pi(N \pi+\sigma t)^{\alpha}}, \quad|t|<N \pi / \sigma
$$


Proof. Since for a fixed $t \in \mathbb{R}$, the function $1 /(u \pi-\sigma t)$ is strictly decreasing for $u>\sigma t / \pi$, then

$$
\sum_{n>N} \frac{1}{(\sigma t-n \pi)^{\alpha+1}}<\int_{N}^{\infty} \frac{d u}{(\sigma t-\pi u)^{\alpha+1}}=\frac{1}{\alpha \pi(N \pi-\sigma t)^{\alpha}}
$$

which is (2.1). Inequality (2.2) can be deduced from (2.1) since the domain of $t$, i.e., $|t|<N \pi / \sigma$, is symmetric about $t=0$.

Lemma 2.2. Let $f(t) \in P W_{\sigma}^{2}$ and $t^{k} f(t) \in L^{2}(\mathbb{R}), k \in \mathbb{Z}^{+}$. Then

$$
\begin{gathered}
\left(\sum_{n>N}\left|f^{\prime}\left(\frac{n \pi}{\sigma}\right)\right|^{2}\right)^{1 / 2} \leq \frac{\xi_{k, \sigma}\left(\sigma E_{k}+k E_{k-1}\right)}{(N+1)^{k}} \\
\left(\sum_{n<-N}\left|f^{\prime}\left(\frac{n \pi}{\sigma}\right)\right|^{2}\right)^{1 / 2} \leq \frac{\xi_{k, \sigma}\left(\sigma E_{k}+k E_{k-1}\right)}{(N+1)^{k}}
\end{gathered}
$$

where

$$
E_{k}:=\sqrt{\int_{-\infty}^{\infty}\left|t^{k} f(t)\right|^{2} d t}, \quad \xi_{k, \sigma}:=\frac{\sigma^{k+1 / 2}}{\pi^{k+1} \sqrt{1-4^{-k}}} .
$$

Proof. Since $f(t) \in P W_{\sigma}^{2}$, then

$$
\left(t^{k} f(t)\right)^{\prime}=t^{k} f^{\prime}(t)+k t^{k-1} f(t), \quad k \geq 1 .
$$

Using Minkowski's and Bernstein's inequalities [18, p. 49], we obtain

$$
\begin{aligned}
& \left(\int_{-\infty}^{\infty}\left|t^{k} f^{\prime}(t)\right|^{2} d t\right)^{1 / 2} \\
\leq & \left(\int_{-\infty}^{\infty}\left|\left(t^{k} f(t)\right)^{\prime}\right|^{2} d t\right)^{1 / 2}+k\left(\int_{-\infty}^{\infty}\left|t^{k-1} f(t)\right|^{2} d t\right)^{1 / 2} \\
\leq & \sigma\left(\int_{-\infty}^{\infty}\left|t^{k} f(t)\right|^{2} d t\right)^{1 / 2}+k\left(\int_{-\infty}^{\infty}\left|t^{k-1} f(t)\right|^{2} d t\right)^{1 / 2} \\
= & \sigma E_{k}+k E_{k-1} .
\end{aligned}
$$

Hence $t^{k} f^{\prime}(t) \in L^{2}(\mathbb{R})$. Now we prove that $t^{k} f^{\prime}(t) \in P W_{\sigma}^{2}$. Let $\mathcal{F}$ denote the continuous Fourier transform. Then, [16, p. 20],

$$
\mathcal{F}\left(t^{k} f^{\prime}(t)\right)(\alpha)=(i)^{k} \mathcal{F}^{(k)}\left(f^{\prime}(t)\right)(\alpha), \quad \alpha \in \mathbb{R} .
$$

Thus the Fourier transform of $t^{k} f^{\prime}(t)$ vanishes outside $]-\sigma, \sigma$, i.e., $f^{\prime}(t) \in$ $P W_{\sigma}^{2}$. Hence, [18, p. 59],

$$
\int_{-\infty}^{\infty} t^{2 k}\left|f^{\prime}(t)\right|^{2} d t=\frac{\pi}{\sigma} \sum_{j=-\infty}^{\infty}\left(\frac{j \pi}{\sigma}\right)^{2 k}\left|f^{\prime}\left(\frac{j \pi}{\sigma}\right)\right|^{2} .
$$


Combining (2.8) and (2.10) we obtain

$$
\frac{\pi}{\sigma} \sum_{j=-\infty}^{\infty}\left(\frac{j \pi}{\sigma}\right)^{2 k}\left|f^{\prime}\left(\frac{j \pi}{\sigma}\right)\right|^{2} \leq\left(\sigma E_{k}+k E_{k-1}\right)^{2} .
$$

Thus for $n \in \mathbb{Z}^{+}$

$$
\frac{\pi}{\sigma} \sum_{j=n}^{2 n-1}\left(\frac{j \pi}{\sigma}\right)^{2 k}\left|f^{\prime}\left(\frac{j \pi}{\sigma}\right)\right|^{2} \leq\left(\sigma E_{k}+k E_{k-1}\right)^{2},
$$

and therefore

$$
\sum_{j=n}^{2 n-1}\left|f^{\prime}\left(\frac{j \pi}{\sigma}\right)\right|^{2} \leq\left(\frac{\sigma}{\pi}\right)^{2 k+1} \frac{\left(\sigma E_{k}+k E_{k-1}\right)^{2}}{n^{2 k}} .
$$

Letting $n:=2^{r}(N+1)$ in $(2.13), N, r \in \mathbb{Z}^{+}$, then

$$
\begin{aligned}
\sum_{j>N}\left|f^{\prime}\left(\frac{j \pi}{\sigma}\right)\right|^{2} & =\sum_{r=0}^{\infty} \sum_{j=2^{r}(N+1)}^{2^{r+1}(N+1)-1}\left|f^{\prime}\left(\frac{j \pi}{\sigma}\right)\right|^{2} \\
& \leq\left(\frac{\sigma}{\pi}\right)^{2 k+1} \frac{\left(\sigma E_{k}+k E_{k-1}\right)^{2}}{(N+1)^{2 k}} \sum_{r=0}^{\infty} \frac{1}{4^{r k}},
\end{aligned}
$$

which is (2.4). The proof of (2.5) can be achieved similarly.

Remark 2.3. According to a referee's suggestion there might be another proof that $t^{k} f(t)$ is of exponential type $\sigma$ whenever $f(t) \in P W_{\sigma}^{2}$ based on the fact that for any polynomial $p(t), p(t) f^{\prime}(t)$ has exponential type $\sigma$.

Lemma 2.4. Suppose that $f(t) \in P W_{\sigma}^{2}$ satisfies a decay condition of the form

$$
|f(t)| \leq \frac{A}{|t|^{\alpha+1}}, \quad t \neq 0,
$$

where $A>0$ and $0<\alpha \leq 1$ are constants. Then for $N \in \mathbb{Z}^{+}$we have

$$
\begin{gathered}
\left(\sum_{k>N}\left|f^{\prime}\left(\frac{k \pi}{\sigma}\right)\right|^{2}\right)^{1 / 2}<\frac{M_{1}(\rho(N)+2)}{\sqrt{N}}+\frac{M_{2}(\rho(2 N)+4)}{N^{\alpha+1 / 2}} \\
\left(\sum_{k<-N}\left|f^{\prime}\left(\frac{k \pi}{\sigma}\right)\right|^{2}\right)^{1 / 2}<\frac{M_{1}(\rho(N)+2)}{\sqrt{N}}+\frac{M_{2}(\rho(2 N)+4)}{N^{\alpha+1 / 2}},
\end{gathered}
$$

where

(2.18) $\quad M_{1}:=\frac{3 \sigma}{\pi}\left(|f(0)|+A\left(\frac{\sigma}{\pi}\right)^{\alpha}\right), M_{2}:=A\left(\frac{\sigma}{\pi}\right)^{\alpha+1}, \rho(t):=\gamma+\log (t)$. 
Proof. Let $f(t) \in P W_{\sigma}^{2}$. Then

$$
f(t)=\sum_{n=-\infty}^{\infty} f\left(\frac{n \pi}{\sigma}\right) S_{n}(t), \quad t \in \mathbb{R},
$$

and

$$
f^{\prime}(t)=\sum_{n=-\infty}^{\infty} f\left(\frac{n \pi}{\sigma}\right) S_{n}^{\prime}(t), \quad t \in \mathbb{R} .
$$

Setting $t=k \pi / \sigma$ in $(2.20)$, we obtain

$$
f^{\prime}\left(\frac{k \pi}{\sigma}\right)=\sum_{n=-\infty}^{\infty} f\left(\frac{n \pi}{\sigma}\right) S_{n}^{\prime}\left(\frac{k \pi}{\sigma}\right) .
$$

A simple calculation yields

$$
S_{n}^{\prime}\left(\frac{k \pi}{\sigma}\right)=\left\{\begin{array}{cc}
\frac{\sigma(-1)^{k-n}}{\pi(k-n)}, & n \neq k, \\
0, & n=k .
\end{array}\right.
$$

Let $f(t)$ satisfy the decay condition (2.15). Then for a positive integer $k$ we have

$$
\begin{aligned}
& \left|f^{\prime}\left(\frac{k \pi}{\sigma}\right)\right| \\
\leq & \frac{\sigma|f(0)|}{k \pi}+\left(\frac{\sigma}{\pi}\right)^{\alpha+1} A \sum_{\substack{n=-\infty \\
0 \neq n \neq k}}^{\infty} \frac{1}{|n|^{\alpha+1}|k-n|} \\
= & \frac{\sigma|f(0)|}{k \pi}+\left(\frac{\sigma}{\pi}\right)^{\alpha+1} A\left(\frac{1}{2 k^{\alpha+2}}+\sum_{n=1}^{k-1} \frac{2 k}{n^{\alpha+1}\left(k^{2}-n^{2}\right)}+\sum_{n=k+1}^{\infty} \frac{2}{n^{\alpha}\left(n^{2}-k^{2}\right)}\right) \\
< & \frac{\sigma|f(0)|}{k \pi}+\left(\frac{\sigma}{\pi}\right)^{\alpha+1} A\left(\frac{1}{2 k^{2}}+\sum_{n=1}^{k-1} \frac{2 k}{n\left(k^{2}-n^{2}\right)}+\frac{1}{k^{\alpha}} \sum_{n=1}^{\infty} \frac{2}{n(n+2 k)}\right) \\
= & \frac{\sigma|f(0)|}{k \pi}+\left(\frac{\sigma}{\pi}\right)^{\alpha+1} \frac{A}{k}\left(\frac{3}{2 k}+\sum_{n=1}^{k-1} \frac{4}{n}-\sum_{n=1}^{2 k-1} \frac{1}{n}+\frac{1}{k^{\alpha}} \sum_{n=1}^{\infty} \frac{2 k}{n(n+2 k)}\right) .
\end{aligned}
$$

Substituting from identities (1.11) into (2.23) yields

$$
\begin{aligned}
\left|f^{\prime}\left(\frac{k \pi}{\sigma}\right)\right| \leq & \frac{\sigma}{\pi}\left(\frac{|f(0)|+(\sigma / \pi)^{\alpha} A(3 \gamma+4 \psi(k)-\psi(2 k))}{k}\right) \\
& +\left(\frac{\sigma}{\pi}\right)^{\alpha+1} \frac{A(\gamma+3 / 2+\psi(2 k+1))}{k^{\alpha+1}} .
\end{aligned}
$$


Use (1.12), (1.13) and (2.24) to get

$$
\left|f^{\prime}\left(\frac{k \pi}{\sigma}\right)\right| \leq \frac{M_{1} \rho(k)}{k}+\frac{M_{2}(\rho(2 k)+2)}{k^{\alpha+1}},
$$

where the positive constants $M_{1}$ and $M_{2}$ and the function $\rho(t)$ are defined in (2.18). Using the triangle and the Cauchy-Schwarz inequalities, implies

$$
\begin{aligned}
& \left(\sum_{k>N}\left|f^{\prime}\left(\frac{k \pi}{\sigma}\right)\right|^{2}\right)^{1 / 2} \\
\leq & M_{1}\left(\sum_{k>N} \frac{\rho^{2}(k)}{k^{2}}\right)^{1 / 2}+\frac{M_{2}}{N^{\alpha}}\left(\sum_{k>N} \frac{\left(\rho^{2}(2 k)+2\right)^{2}}{k^{2}}\right)^{1 / 2} \\
\leq & M_{1}\left(\int_{N}^{\infty} \frac{\rho^{2}(t)}{t^{2}} d t\right)^{1 / 2}+\frac{M_{2}}{N^{\alpha}}\left(\int_{N}^{\infty} \frac{\left(\rho^{2}(2 t)+2\right)^{2}}{t^{2}} d t\right)^{1 / 2} \\
< & \frac{M_{1}\left(\rho^{2}(N)+2\right)}{\sqrt{N}}+\frac{M_{2}\left(\rho^{2}(2 N)+4\right)}{N^{\alpha+1 / 2}}
\end{aligned}
$$

and this proves (2.16). Using similar arguments, we can prove (2.17).

\section{Truncation error}

In the following we will derive estimates for $T(f, N)(t)$. The first result is an analogue of the result of Jagerman [21], and the second is an analogue of Li's [23] for the classical sampling theorem.

Theorem 3.1. Let $f(t) \in P W_{\sigma}^{2}$ and $t^{k} f(t) \in L^{2}(\mathbb{R}), k, N \in \mathbb{Z}^{+}$. Then, for $t \in \mathbb{R},|t|<N \pi / \sigma$, we have

$$
\begin{aligned}
T(f, N)(t) \leq & \frac{\xi_{k, \sigma} E_{k}|\sin \sigma t|^{2}}{\sqrt{3}(N+1)^{k}}\left(\frac{1}{(N \pi-\sigma t)^{3 / 2}}+\frac{1}{(N \pi+\sigma t)^{3 / 2}}\right) \\
& +\frac{\xi_{k, \sigma}\left(\sigma E_{k}+k E_{k-1}\right)|\sin \sigma t|^{2}}{\sigma(N+1)^{k}}\left(\frac{1}{\sqrt{N \pi-\sigma t}}+\frac{1}{\sqrt{N \pi+\sigma t}}\right)
\end{aligned}
$$

where the constants $E_{k}$ and $\xi_{k, \sigma}$ are defined in (2.6).

Proof. Applying the triangle and the Cauchy-Schwarz inequalities to $T(f, N)(t)$ we obtain

$$
\begin{aligned}
T(f, N)(t) \leq & \left(\sum_{n>N}\left|f\left(\frac{n \pi}{\sigma}\right)\right|^{2}\right)^{1 / 2}\left(\sum_{n>N}\left|S_{n}(t)\right|^{4}\right)^{1 / 2} \\
& +\left(\sum_{n<-N}\left|f\left(\frac{n \pi}{\sigma}\right)\right|^{2}\right)^{1 / 2}\left(\sum_{n<-N}\left|S_{n}(t)\right|^{4}\right)^{1 / 2}
\end{aligned}
$$




$$
\begin{aligned}
& +\frac{|\sin \sigma t|}{\sigma}\left(\sum_{n>N}\left|f^{\prime}\left(\frac{n \pi}{\sigma}\right)\right|^{2}\right)^{1 / 2}\left(\sum_{n>N}\left|S_{n}(t)\right|^{2}\right)^{1 / 2} \\
& +\left(\sum_{n<-N}\left|f^{\prime}\left(\frac{n \pi}{\sigma}\right)\right|^{2}\right)^{1 / 2}\left(\sum_{n<-N}\left|S_{n}(t)\right|^{2}\right)^{1 / 2} .
\end{aligned}
$$

Since $f(t) \in P W_{\sigma}^{2}$ and $t^{k} f(t) \in L^{2}(\mathbb{R})$ then using Jagerman's estimations, cf. $[21$, p. 716$]$

$$
\left(\sum_{n>N}\left|f\left(\frac{n \pi}{\sigma}\right)\right|^{2}\right)^{1 / 2} \leq \frac{\xi_{k, \sigma} E_{k}}{(N+1)^{k}}, \quad\left(\sum_{n<-N}\left|f\left(\frac{n \pi}{\sigma}\right)\right|^{2}\right)^{1 / 2} \leq \frac{\xi_{k, \sigma} E_{k}}{(N+1)^{k}},
$$

and (2.4)-(2.5) we obtain for $|t|<N \pi / \sigma$

$$
\begin{aligned}
& T(f, N)(t) \\
\leq & \frac{\xi_{k, \sigma} E_{k}|\sin \sigma t|^{2}}{(N+1)^{k}}\left\{\left(\sum_{n>N} \frac{1}{(\sigma t-n \pi)^{4}}\right)^{1 / 2}+\left(\sum_{n<-N} \frac{1}{(\sigma t-n \pi)^{4}}\right)^{1 / 2}\right\} \\
& +\frac{\xi_{k, \sigma}\left(\sigma E_{k}+k E_{k-1}\right)|\sin \sigma t|^{2}}{\sigma(N+1)^{k}}\left\{\left(\sum_{n>N} \frac{1}{(\sigma t-n \pi)^{2}}\right)^{1 / 2}+\left(\sum_{n<-N} \frac{1}{(\sigma t-n \pi)^{2}}\right)^{1 / 2}\right\} .
\end{aligned}
$$

Substituting from (2.1) and (2.2) into (3.4) with $\alpha=3$ in the first two sums and $\alpha=1$ in the last two ones, we get (3.1).

Theorem 3.2. Suppose that $f(t) \in P W_{\sigma}^{2}$ and satisfies condition $(2.15), 0<$ $\alpha \leq 1$. Then for $t \in \mathbb{R}$ and $N \in \mathbb{Z}^{+}$, we have the estimate

$$
T(f, N)(t) \leq \frac{2 M_{1}(\rho(N)+2)}{\sigma \sqrt{N}}+\frac{2 M_{2}(\rho(2 N)+\sigma+4)}{\sigma N^{\alpha+1 / 2}} .
$$

Moreover if $N \geq 8, N \in \mathbb{Z}^{+}$and $t \in \mathbb{R}$, then we have the refined estimate

$$
T(f, N)(t) \leq \frac{e M_{1} \rho\left(N^{2}\right) \log (N)}{\sigma N}+\frac{e M_{2}\left(\rho\left(2 N^{2}\right)+\sigma+2\right) \log (N)}{\sigma N^{\alpha+1}},
$$

where $M_{1}, M_{2}$ and $\rho(t)$ are defined in (2.18).

Proof. Applying the Cauchy-Schwarz inequality to (1.7) with $p=q=2$ and using (2.16) and (2.17) we obtain for $N \in \mathbb{Z}^{+}$

$$
\begin{aligned}
T(f, N)(t) & \leq \sum_{|n|>N}\left|f\left(\frac{n \pi}{\sigma}\right) S_{n}^{2}(t)\right|+\frac{1}{\sigma} \sum_{|n|>N}\left|f^{\prime}\left(\frac{n \pi}{\sigma}\right) S_{n}(t)\right| \\
& \leq\left\{\left(\sum_{|n|>N}\left|f\left(\frac{n \pi}{\sigma}\right)\right|^{2}\right)^{1 / 2}+\frac{1}{\sigma}\left(\sum_{|n|>N}\left|f^{\prime}\left(\frac{n \pi}{\sigma}\right)\right|^{2}\right)^{1 / 2}\right\}\left(\sum_{|n|>N}\left|S_{n}(t)\right|^{2}\right)^{1 / 2}
\end{aligned}
$$




$$
<\left\{\frac{2 M_{1}(\rho(N)+2)}{\sigma \sqrt{N}}+\frac{2 M_{2}(\rho(2 N)+\sigma+4)}{\sigma N^{\alpha+1 / 2}}\right\}\left(\sum_{|n|>N}\left|S_{n}(t)\right|^{2}\right)^{1 / 2}
$$

where we have used the fact that $\left|S_{n}(t)\right| \leq 1, t \in \mathbb{R}$. Let $p, q>1$ such that $\frac{1}{p}+\frac{1}{q}=1$. Substituting from Splettstößer's inequality, cf. [28],

$$
\left(\sum_{n \in \mathbb{Z}}\left|S_{n}(t)\right|^{q}\right)^{1 / q}<p, \quad t \in \mathbb{R}
$$

in (3.7) with $p=q=2$ we obtain (3.5). Now we prove the estimate in (3.6). From Hölder's inequality, (3.8) and using the fact $\left|S_{n}(t)\right| \leq 1, t \in \mathbb{R}$, we get

$$
\begin{aligned}
T(f, N)(t) & \leq \sum_{|n|>N}\left|f\left(\frac{n \pi}{\sigma}\right) S_{n}^{2}(t)\right|+\frac{1}{\sigma} \sum_{|n|>N}\left|f^{\prime}\left(\frac{n \pi}{\sigma}\right) S_{n}(t)\right| \\
& \leq\left\{\left(\sum_{|n|>N}\left|f\left(\frac{n \pi}{\sigma}\right)\right|^{p}\right)^{1 / p}+\frac{1}{\sigma}\left(\sum_{|n|>N}\left|f^{\prime}\left(\frac{n \pi}{\sigma}\right)\right|^{p}\right)^{1 / p}\right\} p .
\end{aligned}
$$

From (2.15) we obtain

$$
\left(\sum_{n>N}\left|f\left(\frac{n \pi}{\sigma}\right)\right|^{p}\right)^{1 / p} \leq M_{2}\left(\int_{N}^{\infty} \frac{d t}{t^{p(\alpha+1)}}\right)^{1 / p} \leq \frac{M_{2}}{N^{\alpha+1-1 / p}}
$$

Applying the Minkowski inequality and (2.25), implies for $p \geq 2$

$$
\begin{aligned}
& \left(\sum_{n>N}\left|f^{\prime}\left(\frac{n \pi}{\sigma}\right)\right|^{p}\right)^{1 / p} \\
\leq & M_{1}\left(\sum_{k>N} \frac{\rho^{p}(k)}{k^{p}}\right)^{1 / p}+\frac{M_{2}}{N^{\alpha}}\left(\sum_{k>N} \frac{(\rho(2 k)+2)^{p}}{k^{p}}\right)^{1 / p} \\
\leq & \left\{M_{1} \gamma+\frac{M_{2}(\rho(2)+2)}{N^{\alpha}}\right\}\left(\int_{N}^{\infty} \frac{d t}{t^{p}}\right)^{1 / p}+\left\{M_{1}+\frac{M_{2}}{N^{\alpha}}\right\}\left(\int_{N}^{\infty} \frac{\log ^{p}(t)}{t^{p}} d t\right)^{1 / p} \\
< & \left\{M_{1} \gamma+\frac{M_{2}(\rho(2)+2)}{N^{\alpha}}\right\} \frac{N^{1 / p}}{N}+\left\{M_{1}+\frac{M_{2}}{N^{\alpha}}\right\}\left(\int_{N}^{\infty} \frac{\log ^{p}(t)}{t^{p}} d t\right)^{1 / p} .
\end{aligned}
$$

Now we estimate the last integral in (3.11). Let $n_{0}:=\lfloor p\rfloor+1$. Hence

$$
\begin{aligned}
\int_{N}^{\infty} \frac{\log ^{p}(t)}{t^{p}} d t & =\int_{\log (N)}^{\infty} t^{p} e^{-(p-1) t} d t \\
& =N^{1-p}\left\{\sum_{k=0}^{n_{0}-2} \log ^{p-k}(N)\right\}+\int_{\log (N)}^{\infty} t^{p-\lfloor p\rfloor} e^{-(p-1) t} d t
\end{aligned}
$$




$$
\begin{aligned}
& \leq N^{1-p}\left\{\sum_{k=0}^{n_{0}-2} \log ^{p-k}(N)\right\}+\int_{\log (N)}^{\infty} t e^{-(p-1) t} d t \\
& \leq(p+1) N^{1-p} \log ^{p}(N) .
\end{aligned}
$$

Substituting from (3.12) into (3.11) and noting that the function $(1+t)^{1 / t}$, $t \geq 2$ is decreasing, we obtain for $p \geq 2$

$$
\left(\sum_{k>N}\left|f^{\prime}\left(\frac{k \pi}{\sigma}\right)\right|^{p}\right)^{1 / p}<\frac{M_{1} \rho\left(N^{2}\right) N^{1 / p}}{N}+\frac{M_{2}\left(\rho\left(2 N^{2}\right)+2\right) N^{1 / p}}{N^{\alpha+1}} .
$$

Combining (3.13), (3.9) and (3.10), yields for $p \geq 2$

$$
T(f, N)(t)<\left\{\frac{2 M_{1} \rho\left(N^{2}\right) N^{1 / p}}{\sigma N}+\frac{2 M_{2}\left(\rho\left(2 N^{2}\right)+\sigma+2\right) N^{1 / p}}{\sigma N^{\alpha+1}}\right\} p .
$$

In (3.14) choosing $N>e^{2}$ and $p:=\log (N)$ completes the proof since $N^{1 / p}=$ e.

\section{Amplitude and jitter errors}

In this section we study the amplitude and jitter errors associated with the sampling series of Hermite type (1.6).

Theorem 4.1. Let $f(t) \in P W_{\sigma}^{2}$ satisfy the condition (2.15) and

$$
\left|\varepsilon_{n}\right| \leq|f(n \pi / \sigma)|, \quad\left|\varepsilon_{n}^{\prime}\right| \leq\left|f^{\prime}(n \pi / \sigma)\right|, \quad n \in \mathbb{Z} .
$$

Then for $0<\varepsilon \leq \min \{\pi / \sigma, \sigma / \pi, 1 / \sqrt{e}\}$, we have

$$
\begin{aligned}
\|\mathcal{A}(\varepsilon, f)\|_{\infty} \leq \frac{4 e^{1 / 4}}{\sigma(\alpha+1)}\{ & \sqrt{3} e(1+\sigma)+(\pi / \sigma) M_{1} \rho\left(\varepsilon^{-10}\right) \\
& \left.+A\left(\rho\left(2 \varepsilon^{-10}\right)+\sigma+2\right)\right\} \varepsilon \log (1 / \varepsilon) .
\end{aligned}
$$

Proof. Let $p, q>1$ such that $\frac{1}{p}+\frac{1}{q}=1$. From Hölder's inequality and using (3.8), the amplitude error defined in (1.9) satisfies

$$
\begin{aligned}
|\mathcal{A}(\varepsilon, f)(t)| & \leq\left(\sum_{n=-\infty}^{\infty}\left|S_{n}(t)\right|^{2 q}\right)^{1 / q}\left(\sum_{n=-\infty}^{\infty}\left|\varepsilon_{n}\right|^{p}\right)^{1 / p}+\frac{1}{\sigma}\left(\sum_{n=-\infty}^{\infty}\left|S_{n}(t)\right|^{q}\right)^{1 / q}\left(\sum_{n=-\infty}^{\infty}\left|\varepsilon_{n}^{\prime}\right|^{p}\right)^{1 / p} \\
(4.3) & <\left\{\left(\sum_{n=-\infty}^{\infty}\left|\varepsilon_{n}\right|^{p}\right)^{1 / p}+\frac{1}{\sigma}\left(\sum_{n=-\infty}^{\infty}\left|\varepsilon_{n}^{\prime}\right|^{p}\right)^{1 / p}\right\} p, \quad t \in \mathbb{R} .
\end{aligned}
$$

Now we estimate the infinite sums above. Applying the Minkowski inequality, we obtain for $N \geq 1$

$$
\left(\sum_{n=-\infty}^{\infty}\left|\varepsilon_{n}\right|^{p}\right)^{1 / p} \leq\left(\sum_{|n| \leq N}\left|\varepsilon_{n}\right|^{p}\right)^{1 / p}+\left(\sum_{|n|>N}\left|\varepsilon_{n}\right|^{p}\right)^{1 / p} .
$$


Using (2.15), (4.1) and choosing $p \geq 2$, yield

$$
\left(\sum_{|n|>N}\left|\varepsilon_{n}\right|^{p}\right)^{1 / p} \leq\left(\sum_{|n|>N}\left|f\left(\frac{n \pi}{\sigma}\right)\right|^{p}\right)^{1 / p} \leq \frac{M_{2}}{N^{\alpha+1-1 / p}} .
$$

Moreover,

$$
\left(\sum_{n=-N}^{N}\left|\varepsilon_{n}\right|^{p}\right)^{1 / p} \leq \varepsilon(2 N+1)^{1 / p} .
$$

Combining (4.4)-(4.5), we obtain for $N \geq 1, p \geq 2$

$$
\left(\sum_{n=-\infty}^{\infty}\left|\varepsilon_{n}\right|^{p}\right)^{1 / p}<\varepsilon(2 N+1)^{1 / p}+\frac{M_{2}}{N^{\alpha+1-1 / p}} .
$$

Since $f(t)$ satisfies condition (2.15), then from (4.1) and (3.13), we get for $N \geq 1, p \geq 2$

$$
\left(\sum_{|n|>N}\left|\varepsilon_{n}^{\prime}\right|^{p}\right)^{1 / p}<\frac{M_{1} \rho\left(N^{2}\right) N^{1 / p}}{N}+\frac{M_{2}\left(\rho\left(2 N^{2}\right)+2\right) N^{1 / p}}{N^{\alpha+1}} .
$$

Substituting from (4.7) and (4.6) into (4.3) we end with

$$
|\mathcal{A}(\varepsilon, f)|<\left\{\frac{\varepsilon(1+\sigma)(2 N+1)^{1 / p}}{\sigma}+\frac{M_{1} \rho\left(N^{2}\right)}{\sigma N^{1-1 / p}}+\frac{M_{2}\left(\rho\left(2 N^{2}\right)+\sigma+2\right)}{\sigma N^{\alpha+1-1 / p}}\right\} p .
$$

The parameters $N$ and $p$ can to be chosen suitably to obtain the desired estimate. We distinguish between two cases. First when $\sigma \geq \pi$ we take

$$
N:=\left\lfloor\varepsilon^{-1 /(\alpha+1)}\left(\frac{\sigma}{\pi}\right)^{\frac{(\alpha+1) p}{(\alpha+1) p-1}}\right\rfloor, \quad p=\frac{4}{\alpha+1} \log (1 / \varepsilon) .
$$

Since $\varepsilon \leq\{\pi / \sigma, 1 / \sqrt{e}\}$, then $\frac{\sigma}{\pi} \leq \frac{1}{\varepsilon}$ and $N \geq 1$. Therefore

$$
\begin{aligned}
(2 N+1)^{1 / p} & \leq \sqrt{3}\left(\frac{1}{\varepsilon}\right)^{\frac{3(\alpha+1) p-1}{(\alpha+1) p((\alpha+1) p-1)}} \leq \sqrt{3}\left(\frac{1}{\varepsilon}\right)^{5 /(\alpha+1) p}=\sqrt{3} e^{5 / 4} \\
N^{\frac{1-(1+\alpha) p}{p}} & \leq\left(\frac{\pi}{\sigma}\right)^{\alpha+1} e^{1 / 4} \varepsilon \\
\rho\left(N^{2}\right) & \leq \gamma+10 \log (1 / \varepsilon) .
\end{aligned}
$$

Substituting from (4.10) into (4.8) and noting that $p=\frac{4}{\alpha+1} \log (1 / \varepsilon)$, we obtain (4.2). If $\sigma<\pi$ we choose

$$
N:=\left\lfloor\varepsilon^{-1 /(\alpha+1)}\left(\frac{\pi}{\sigma}\right)^{\frac{(\alpha+1) p}{(\alpha+1) p-1}}\right\rfloor
$$

and proceed as in the previous case. 
In the previous result, as well as in the next one, we use the technique of Butzer et al. [10, 14, 15]. Now we study the time-jitter error associated with (1.6) where the decay condition (2.15) is satisfied.

Theorem 4.2. Let $f(t) \in P W_{\sigma}^{2}$ be such that condition (2.15) holds. Then

$$
\begin{aligned}
\|J(\delta, f)\|_{\infty} \leq(\sqrt{5} & e\left(\sigma\left\|f^{\prime}\right\|_{\infty}+\left\|f^{\prime \prime}\right\|_{\infty}\right)+2 \sigma \sqrt{2} A \\
& +\frac{4 \pi e^{1 / 4} M_{1} \rho\left(1 / \delta^{10}\right)}{\sigma} \\
& \left.\left.+4 A\left(\rho\left(2 / \delta^{10}\right)+2\right)\right) \frac{4 \delta \log (1 / \delta)}{\sigma(\alpha+1)}\right),
\end{aligned}
$$

provided that $\delta \leq \min \{\pi / \sigma, \sigma / \pi, 1 / \sqrt{e}\}$.

Proof. Let $p, q>1$ such that $\frac{1}{p}+\frac{1}{q}=1$. Applying Hölder's inequality for (1.10) and using Splettstößer's inequality (3.8), we obtain

$$
\begin{aligned}
|J(\delta, f)(t)| \leq & \left(\sum_{n=-\infty}^{\infty}\left|f\left(\frac{n \pi}{\sigma}\right)-f\left(\frac{n \pi}{\sigma}+\delta_{n}\right)\right|^{p}\right)^{1 / p}\left(\sum_{n=-\infty}^{\infty}\left|S_{n}(t)\right|^{2 q}\right)^{1 / q} \\
& +\frac{1}{\sigma}\left(\sum_{n=-\infty}^{\infty}\left|f^{\prime}\left(\frac{n \pi}{\sigma}\right)-f^{\prime}\left(\frac{n \pi}{\sigma}+\delta_{n}\right)\right|^{p}\right)^{1 / p}\left(\sum_{n=-\infty}^{\infty}\left|S_{n}(t)\right|^{q}\right)^{1 / q} .
\end{aligned}
$$

Applying the mean value theorem, Minkowski inequality and (2.15) to (4.13) yield

$$
\left(\sum_{n=-\infty}^{\infty}\left|f\left(\frac{n \pi}{\sigma}\right)-f\left(\frac{n \pi}{\sigma}+\delta_{n}\right)\right|^{p}\right)^{1 / p} \leq \sqrt{5} e \delta\left\|f^{\prime}\right\|_{\infty}+2 \sqrt{2} A \delta e^{1 / 4}
$$

for $\sigma \geq \pi$, cf. [10, 18, pp. 116-117]. Also inequality (4.6) holds for $\sigma<\pi$, see [3]. Using the mean value theorem, $N \geq 1$, we obtain

$$
\begin{aligned}
& \left(\sum_{|n| \leq N}\left|f^{\prime}\left(\frac{n \pi}{\sigma}\right)-f^{\prime}\left(\frac{n \pi}{\sigma}+\delta_{n}\right)\right|^{p}\right)^{1 / p} \\
= & \left(\sum_{|n| \leq N}\left|f^{\prime \prime}\left(t_{n}\right) \delta_{n}\right|^{p}\right)^{1 / p} \leq \delta\left\|f^{\prime \prime}\right\|_{\infty}(2 N+1)^{1 / p},
\end{aligned}
$$


where $t_{n} \in\left[\frac{n \pi}{\sigma}, \frac{n \pi}{\sigma}+\delta_{n}\right],|n| \leq N$. The use of the Minkowski inequality, (2.15) and (3.13) leads to

$$
\begin{aligned}
& \left(\sum_{|n|>N}\left|f^{\prime}\left(\frac{n \pi}{\sigma}\right)-f^{\prime}\left(\frac{n \pi}{\sigma}+\delta_{n}\right)\right|^{p}\right)^{1 / p} \\
\leq & \left(\sum_{|n|>N}\left|f^{\prime}\left(\frac{n \pi}{\sigma}\right)\right|^{p}\right)^{1 / p}+\left(\sum_{|n|>N}\left|f^{\prime}\left(\frac{n \pi}{\sigma}+\delta_{n}\right)\right|^{p}\right)^{1 / p} \\
< & \frac{4 M_{1} \rho\left(N^{2}\right) N^{1 / p}}{N}+\frac{4 M_{2}\left(\rho\left(2 N^{2}\right)+2\right) N^{1 / p}}{N^{\alpha+1}},
\end{aligned}
$$

where we have used the same calculations as in the proof of Theorem 3.2 to estimate the second sum of (4.16). Combining (4.13)-(4.16) and using (3.8) we arrive at

$$
\begin{aligned}
|J(\delta, f)(t)|<( & \sqrt{5} e \delta\left\|f^{\prime}\right\|_{\infty}+2 \sqrt{2} A \delta e^{1 / 4}+\frac{\delta\left\|f^{\prime \prime}\right\|_{\infty}(2 N+1)^{1 / p}}{\sigma} \\
& \left.+\frac{4 M_{1} \rho\left(N^{2}\right)}{\sigma N^{1-1 / p}}+\frac{4 M_{2}\left(\rho\left(2 N^{2}\right)+2\right)}{\sigma N^{\alpha+1-1 / p}}\right) p
\end{aligned}
$$

If $\sigma \geq \pi$ we choose

$$
N:=\left\lfloor\delta^{-1 /(\alpha+1)}\left(\frac{\sigma}{\pi}\right)^{\frac{(\alpha+1) p}{(\alpha+1) p-1}}\right\rfloor, \quad p=\frac{4}{\alpha+1} \log (1 / \delta) .
$$

Therefore, as in (4.10),

$$
\begin{aligned}
(2 N+1)^{1 / p} & \leq \sqrt{3} e^{5 / 4}, \\
N^{\frac{1-(1+\alpha) p}{p}} & \leq\left(\frac{\pi}{\sigma}\right)^{\alpha+1} e^{1 / 4} \delta, \\
\rho\left(N^{2}\right) & \leq \gamma+10 \log (1 / \delta) .
\end{aligned}
$$

Substituting from (4.19) in (4.17), we obtain (4.12). When $\sigma<\pi$ we take

$$
N:=\left\lfloor\delta^{-1 /(\alpha+1)}\left(\frac{\pi}{\sigma}\right)^{\frac{(\alpha+1) p}{(\alpha+1) p-1}}\right\rfloor .
$$

This completes the proof.

\section{Comparisons and prospectives}

In this section we give some examples and comparisons indicating that the use of Hermite interpolation formula (1.6) in approximation theory may be better than the use of the classical sampling formula

$$
f(t)=\sum_{n=-\infty}^{\infty} f\left(\frac{n \pi}{\sigma}\right) S_{n}(t), \quad t \in \mathbb{R}, f \in P W_{\sigma}^{2} .
$$


The use of (1.6) makes no restriction on $f$ since $f$ is entire and $P W_{\sigma}^{2} \subseteq P W_{2 \sigma}^{2}$. We consider in the following two examples two functions and sketch the graph of each function $f$ and its approximations. We denote by $f_{L, N}$ and $f_{H, N}$ the classical sampling and the Hermite approximations respectively. We also give tables illustrating the error for some numerical values, where $E_{L}$ and $E_{H}$ denote the absolute errors in the use of (1.6) and (5.1) respectively.

Example 5.1. The function

$$
f(t)=\frac{-\sqrt{2 \pi} \cos (\pi t)}{t}+\frac{\sqrt{2} \sin (\pi t)}{\sqrt{\pi} t^{2}},
$$

is a $P W_{\pi}^{2} \subseteq P W_{2 \pi}^{2}$ function. Let $f_{L, 7}(t)$ and $f_{H, 3}(t)$ denote the truncated series

$$
\begin{gathered}
f_{L, 7}(t)=\sum_{n=-7}^{7} f(n) \frac{\sin (\pi t-n \pi)}{(\pi t-n \pi)}, \\
f_{H, 3}(t)=\sum_{n=-3}^{3} f(n)\left(\frac{\sin (\pi t-n \pi)}{(\pi t-n \pi)}\right)^{2}+f^{\prime}(n) \frac{(\sin (\pi t-n \pi))^{2}}{\pi(\pi t-n \pi)} .
\end{gathered}
$$

Note that in (5.3) we have the 7-th iteration with 15 terms, while in (5.4) we have the third one with 14 terms. Figures 1,2 show $f$ and each approximation.

Example 5.2. Let us consider the $P W_{2}^{2}$-function

$$
g(t)=\frac{\sqrt{2}(\cos (2) \sin (2 t)-t \sin (2) \cos (2 t))}{\sqrt{\pi}\left(t^{2}-1\right)} .
$$

We also consider the iterations $g_{L, 7}(t)$ and $g_{H, 3}(t)$ as

$$
\begin{gathered}
g_{L, 7}(t)=\sum_{n=-7}^{7} g\left(\frac{n \pi}{2}\right) \frac{\sin (2 t-n \pi)}{(2 t-n \pi)} \\
g_{H, 3}(t)=\sum_{n=-3}^{3} g\left(\frac{n \pi}{2}\right)\left(\frac{\sin (2 t-n \pi)}{(2 t-n \pi)}\right)^{2}+g^{\prime}\left(\frac{n \pi}{2}\right) \frac{(\sin (2 t-n \pi))^{2}}{2(2 t-n \pi)} .
\end{gathered}
$$

Also Figures 3, 4 illustrate $g$ and its approximations.

\begin{tabular}{|l|l|l|l|l|l|}
\hline$t_{n}$ & $f\left(t_{n}\right)$ & $f_{L, 15}\left(t_{n}\right)$ & $f_{H, 7}\left(t_{n}\right)$ & $E_{L}$ & $E_{H}$ \\
\hline 0.6 & 3.39885486685528 & 3.3009259560631783 & 3.3990234538820974 & 0.0979289 & 0.000168587 \\
\hline 1.2 & 1.36423685377562 & 1.4248511452262034 & 1.3643693050612276 & 0.06061430 & 0.000132451 \\
\hline 1.8 & -1.27136220643049 & -1.210595323778491 & -1.2711537434789448 & 0.06076688 & 0.000208463 \\
\hline 2.4 & -0.19100424553078 & -0.289676574573938 & -0.1902235362831473 & 0.09867233 & 0.000780709 \\
\hline
\end{tabular}

\begin{tabular}{|l|l|l|l|l|l|}
\hline$t_{n}$ & $g\left(t_{n}\right)$ & $g_{L, 15}\left(t_{n}\right)$ & $g_{H, 7}\left(t_{n}\right)$ & $E_{L}$ & $E_{H}$ \\
\hline 0.5 & 0.6338642152580218 & 0.6178955646599543 & 0.6338670177287358 & 0.01596865 & 0.00000280 \\
\hline 1.5 & 0.8244188818883641 & 0.8217378230412181 & 0.8244554296153525 & 0.00268109 & 0.00003655 \\
\hline 2.5 & -0.0373531457979486 & -0.019093862328041 & -0.0373310336058788 & 0.01825928 & 0.00002211 \\
\hline 3.5 & -0.1895580610349899 & -0.202110823670597 & -0.18954728942624 & 0.01255276 & 0.00001077 \\
\hline
\end{tabular}




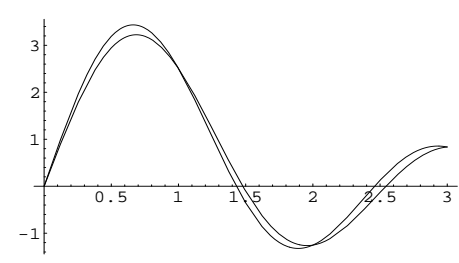

Figure 1. $f_{L, 7}(t)$ and $f(t)$

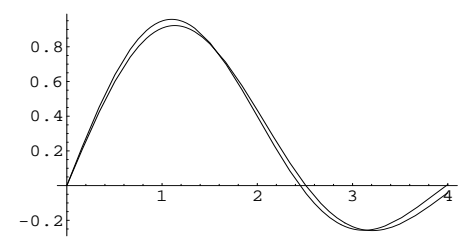

FiguRE 3. $g_{L, 7}(t)$ and $g(t)$

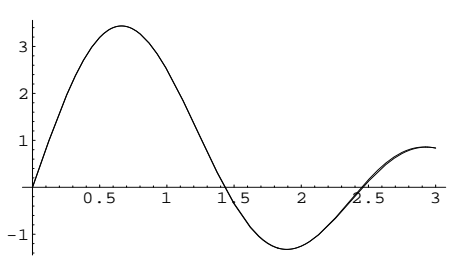

Figure 2. $f_{H, 3}(t)$ and $f(t)$

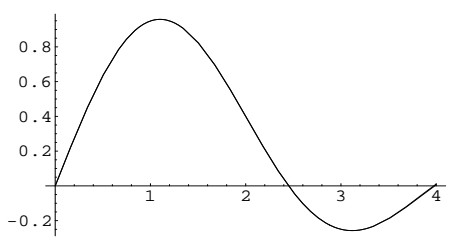

FiguRE $4 . g_{H, 3}(t)$ and $g(t)$

The estimates derived in Sections 3, 4 above can be used in numerical approximation of several mathematical problems. For example it can be used to find error bounds for the computations of eigenvalues of differential operators as is done in $[4,5,6,8]$ by the use of (5.1). For a general application of the method to compute eigenvalues, one has to extend the results to compute error estimates associated with (1.6) on $\mathbb{C}$ and to compute the error estimates for the derivative of (1.6) too. Moreover, we may use higher order Hermite interpolation of the type, cf. [20],

$$
f(t)=\sum_{n=-\infty}^{\infty} \sum_{i=0}^{r} f^{(i)}\left(\frac{n \pi}{\sigma}\right) \Psi_{r, n, i}(t)
$$

where

$$
\Psi_{r, n, i}(t):=\left.\sum_{j=0}^{i-1} \frac{G^{r+1}(t)}{\left(t-\frac{n \pi}{\sigma}\right) i !(r-i-j) !}\left\{\left(\frac{\left(t-\frac{n \pi}{\sigma}\right)}{G(t)}\right)^{r+1}\right\}^{(r-i-j)}\right|_{n \pi / \sigma}
$$

and

$$
G(t)=t \prod_{n=1}^{\infty}\left(1-\left(\frac{\sigma t}{n \pi}\right)^{2}\right)
$$

for $f \in P W_{(r+1) \sigma}^{2}$ and $r$ is a fixed positive integer. In this situation one should first obtain error estimates of the type (3.1), (4.1) and (4.12) for (5.8).

Acknowledgement. The authors thank the referee for the constructive comments. The first author is on leave from Department of Mathematics, Faculty of Science, Cairo University, Giza, Egypt. 


\section{References}

[1] M. Abramowitz and I. A. Stegun, Handbook of Mathematical Functions with Formulas, Graphs and Mathematical Tables, Dover, New York, 1972.

[2] H. Alzer, On some inequalities for the gamma and psi functions, Math. Comp. 66 (1997), no. 217, 373-389.

[3] M. H. Annaby and R. M. Asharabi, Truncation, amplitude and jitter errors on $\mathbb{R}$ for sampling series derivatives, Submitted.

[4] _ Approximating eigenvalues of discontinuous problems by sampling theorems, J. Numer. Math. 16 (2008), no. 3, 163-183.

[5] - On sinc-based method in computing eigenvalues of boundary-value problems, SIAM J. Numer. Anal. 46 (2008), no. 2, 671-690.

[6] M. H. Annaby and M. M. Tharwat, On computing eigenvalues of second-order linear pencils, IMA J. Numer. Anal. 27 (2007), no. 2, 366-380.

[7] R. P. Boas, Entire Functions, Academic Press, New York, 1954.

[8] A. Boumenir and B. Chanane, Eigenvalues of S-L systems using sampling theory, Appl. Anal. 62 (1996), no. 3-4, 323-334.

[9] J. L. Brown, Jr., Bounds for truncation error in sampling expansions of band-limited signals, IEEE Trans. Information Theory IT-15 (1969), 440-444.

[10] P. L. Butzer, A survey of the Whittaker-Shannon sampling theorem and some of its extensions, J. Math. Res. Exposition 3 (1983), no. 1, 185-212.

[11] P. L. Butzer, W. Engels, and U. Scheben, Magnitude of the truncation error in sampling expansions of band-limited signals, IEEE Trans. Acoust. Speech Signal Process ASSP30 (1982), 906-912.

[12] P. L. Butzer, J. R. Higgins, and R. L. Stens, Sampling theory of signal analysis, Development of mathematics 1950-2000, 193-234, Birkhauser, Basel, 2000.

[13] P. L. Butzer, G. Schmeisser, and R. L. Stens, An introduction to sampling analysis, Nonuniform sampling, 17-121, Inf. Technol. Transm. Process. Storage, Kluwer/Plenum, New York, 2001.

[14] P. L. Butzer and W. Splettstösser, On quantization, truncation and jitter errors in the sampling theorem and its generalizations, Signal Process. 2 (1980), no. 2, 101-112.

[15] P. L. Butzer, W. Splettstösser, and R. L. Stens, The sampling theorem and linear prediction in signal analysis, Jahresber. Deutsch. Math.-Verein. 90 (1988), no. 1, 1-70.

[16] K. Chandrasekharan, Classical Fourier Transforms, Springer-Verlag, Berlin, 1989.

[17] G. R. Grozev and Q. I. Rahman, Reconstruction of entire functions from irregularly spaced sample points, Canad. J. Math. 48 (1996), no. 4, 777-793.

[18] J. R. Higgins, Sampling Theory in Fourier and Signal Analysis Foundations, Oxford University Press, Oxford, 1996.

[19] J. R. Higgins, G. Schmeisser, and J. J. Voss, The sampling theorem and several equivalent results in analysis, J. Comput. Anal. Appl. 2 (2000), no. 4, 333-371.

[20] G. Hinsen, Irregular sampling of bandlimited $L^{p}$-functions, J. Approx. Theory 72 (1993), no. 3, 346-364

[21] D. Jagerman, Bounds for truncation error of the sampling expansion, SIAM J. Appl. Math. 14 (1966), 714-723.

[22] D. Jagerman and L. Fogel, Some general aspects of the sampling theorem, IRE Trans. Inform. Theory 2 (1956), 139-146.

[23] X. M. Li, Uniform bounds for sampling expansions, J. Approx. Theory 93 (1998), no. $1,100-113$.

[24] J. Lund and K. Bowers, Sinc Methods for Quadrature and Differential Equations, SIAM, Philadelphia, 1992

[25] H. Minc and L. Sathre, Some inequalities involving $(r !)^{1 / r}$, Proc. Edinburgh Math. Soc. (2) $14(1964 / 1965), 41-46$. 
[26] R. Paley and N. Wiener, Fourier Transforms in the Complex Domain, Amer. Math. Soc. Colloq. Publ 19, 1934.

[27] H. S. Piper, Jr., Bounds for truncation error in sampling expansios of finite energy band-limited signals, IEEE Trans. Inform. Theory IT-21 (1975), 482-485.

[28] W. Splettstösser, R. L. Stens, and G. Wilmes, On approximation by the interpolating series of G. Valiron, Funct. Approx. Comment. Math. 11 (1981), 39-56.

[29] F. Stenger, Numerical methods based on Whittaker cardinal, or sinc functions, SIAM Rev. 23 (1981), no. 2, 165-224.

[30] - Numerical Methods Based on Sinc and Analytic Functions, Springer-Verlag, Berlin, 1993

[31] J. J. Voss, Irreguläres Abtasten: Fehleranalyse, Anwendungen und Erweiterungen, Ph. D. thesis, Erlangen, 1999

[32] K. Yao and J. B. Thomas, On truncation error bounds for sampling representations of band-limited signals, IEEE Trans. Aerospace Electronic Syst. AES-2 (1966), 640-647.

Mahmoud H. Annaby

Department of Mathematics

Statistics \& Physics

QATAR University

P. O. Box 2713 Doha, QATAR

E-mail address: mannaby@qu.edu.qa, mhannaby@yahoo.com

Rashad M. Asharabi

Department of Mathematics

FACUlTy of SCIENCE

SANA'A UNIVERSITY

YEMEN

E-mail address: rashad1974@hotmail.com 